\title{
DemDx: An innovative digital clinical decision support software for medical students
}

\author{
Authors: Mala Mawkin, ${ }^{\mathrm{A}}$ Kiran Patel, ${ }^{\mathrm{A}}$ Alice Lee, ${ }^{\mathrm{A}}$ Karen Aspey, ${ }^{\mathrm{A}}$ Thomas MacKinnon ${ }^{\mathrm{A}}$ and Lorin Gresser ${ }^{\mathrm{B}, \mathrm{C}}$
}

\section{Aims}

DemDx has had 23,148 downloads in 118 countries since November 2016, and this study aimed to examine its use as a tool to support the process of clinical decision making for medical students.

\section{Methods}

Thirty medical undergraduates at King's College London were recruited to take part in teaching sessions that utilised DemDx. The teaching sessions lasted approximately 90 minutes and involved a paediatric consultant taking the students through a set of cases referring to DemDx as a prompt to explain the stepby-step clinical reasoning process of history taking, examination results and investigations. The students were then asked to complete an anonymous web-based questionnaire.

\section{Results}

The results suggested that DemDx enhanced the learning of the clinical decision process. $97 \%$ of participants enjoyed using the app. $100 \%$ agreed that DemDx helped them to understand important clinical symptoms and signs to reach diagnosis. $93 \%$ of participants agreed that DemDx helped them to understand key investigations and how to interpret the results. $83 \%$ of participants said DemDx guided them to reach relevant differentials. All 30 students would use the app again to aid in understanding clinical decision making during teaching sessions, on the ward and when revising.

\section{Conclusion}

DemDx provided an innovative, versatile and unique tool that seems to be effective for clinical reasoning training. There is currently no similar tool available to aid students from the classroom to bedside when training in clinical reasoning; the app guides the user from the presenting symptoms through to a diagnosis, enabling medical students to take a more structured and reasoned approach to clinical care, avoiding unnecessary

Authors: ${ }^{A}$ Department of Medicine, Imperial College London ${ }^{B}$ DemDx; ' Department of Medicine, King's College London, London, UK investigations, treatment and missed diagnoses. Further research is now needed in the medical schools that are utilising DemDx in countries such as USA, Brazil and Kenya to understand, and thus optimise, student engagement to ensure medical students feel prepared for clinical decision making globally.

\section{Conflict of interest statement}

This paper was co-authored by medical undergraduates, and information regarding app statistics provided by the team at DemDx. 